\title{
Contribuição para análise dos custos no tratamento da água utilizando programação linear fuzzy: um modelo para o gerenciamento do abastecimento do município de Campos (RJ)
}

Contribution to cost analysis in water treatment using fuzzy linear programming: a model for the supply management in the city of Campos, Rio de Janeiro, Brazil

\section{Leonardo do Espírito Santo Francisco"*, Gudelia Morales de Arica ${ }^{2}$}

\begin{abstract}
RESUMO
A água é um dos recursos naturais essenciais à sobrevivência humana, porém tem sofrido com problemas como sobrecarga de utilização, em âmbito global; distribuição desigual; e escassez, o que contribui negativamente com a disponibilidade do recurso, evidenciando, assim, a crise da água. Nesse cenário, surge então a necessidade de se gerenciar de maneira eficaz esse recurso que é tão importante e que apresenta fortes componentes de incertezas. Um importante objeto do gerenciamento é o tratamento químico pelo qual a água bruta passa para se tornar tratada e, então, adequada para o consumo - este processo corresponde ao segundo maior custo nas estações de tratamento de água, atrás apenas de despesas envolvendo recursos humanos, materiais e serviços. Sendo assim, o presente trabalho propõe um modelo de programação linear que incorpora a lógica fuzzy, uma ferramenta eficiente em modelos com incertezas, para auxiliar no gerenciamento de abastecimento de água, considerando os custos com produtos químicos usados no tratamento desse recurso no município de Campos dos Goytacazes. Este modelo foi resolvido utilizando o ambiente Rstudio e apresentou uma redução dos custos em 9,8\% em relação ao modelo de programação linear padrão preparado para o sistema. Conclui-se, então, que o trabalho desenvolvido serve de ponto de partida para uma análise mais minuciosa sobre o impacto dos custos de alocação de água tratada no município e contribui positivamente para a racionalização da água bruta.
\end{abstract} Palavras-chave: gerenciamento de custos; tratamento de água; programação linear; otimização fuzzy em R.

\begin{abstract}
Water is one of the essential natural resources for human survival, but it has undergone problems such as: use overload at global level; unequally distribution; and scarcity that contributes negatively to the availability of the resource, thus evidencing a water crisis. Thus, there is a need to effectively manage this important resource, which presents strong components of uncertainties. An important management object is the chemical treatment through which raw water passes in order to be treated and then appropriate for consumption, because it corresponds to the second largest cost in water treatment plants, only after expenses involving human resources, materials, and services. Thus, the present work proposes a linear programming model that incorporates the fuzzy logic, which is an efficient tool in models with uncertainties to assist in any water supply management, considering costs with chemicals used in the treatment of this resource in the city of Campos dos Goytacazes, Brazil. This model was solved using the Rstudio environment and presented a 9.8\% cost reduction compared with the standard linear programming model prepared for the system. In conclusion, the developed work serves as a starting point for a more detailed analysis of the impact of the costs of treated water allocation in the municipality and contributes positively to the raw water rationalization.
\end{abstract}

Keywords: cost management; water treatment; linear programming; fuzzy optimization in R.

Professor do Instituto Federal Fluminense - Campos dos Goytacazes (RJ), Brasil. 


\section{INTRODUÇÃO}

Um dos recursos naturais essenciais à sobrevivência humana e da maior parte dos seres vivos é a água. Esta, como outros recursos, tem sofrido uma sobrecarga de utilização em nível global. Isso se deve ao fato de a água ser utilizada não somente para atender às necessidades básicas da população, mas também para uma série de atividades, incluindo processos industriais. Segundo o World Wide Fund for Nature - WWF (2014), 45\% da água doce consumida em países industrializados é utilizada na geração de energia. Outro dado relevante é que $70 \%$ do consumo de água doce é utilizado na produção mundial de alimentos.

Sabe-se que a água é um bem comum e indispensável a todos. Sendo assim, esse recurso deve ser gerenciado de forma racional (RODELLA, 2014). A grande questão é que esse recurso não está presente em todas as regiões do planeta e sua distribuição não é feita de maneira uniforme, gerando perdas na distribuição de água realizada pelos prestadores de serviços - o índice médio de perdas na distribuição, em 2014, foi de 37,7\%, por exemplo (SNIS, 2016). E mais: projeções indicam que a demanda global por água doce excederá em $40 \%$ a oferta em 2030 (WWF, 2014), configurando-se uma situação chamada de estresse hídrico, como é o caso de países do Oriente Médio e da África.

Para Tundisi (2008), vivemos uma "crise da água", motivada, principalmente, pela escassez, que pode ser oriunda de alterações na disponibilidade, no aumento da demanda, além de períodos intensos de seca, evidenciando assim a presença de incertezas. Sendo assim, é fundamental que o setor de abastecimento público, que é um grande usuário de águas, comprometa-se em utilizar com maior racionalidade esse recurso, garantindo que todos os demandantes recebam água de maneira uniforme, o que se caracteriza como um grande desafio para seus gestores (ACSELRAD; AZEVEDO; JOHNSSON, 2015; CHAIB et al., 2015).

Propiciar um bom gerenciamento da água trará consigo a diminuição dos seguintes problemas: perdas nos sistemas de abastecimento; consumo irresponsável dos usuários de água tratada; poluição da água; ausência de consciência ambiental; e altos custos de operação e manutenção do sistema de abastecimento público (PAULINO, 2005).

Carvalho et al. (2009) ressaltam que o problema de gerenciamento da água bruta está relacionado à complexidade gerada por fatores como incertezas hidrológicas, incertezas da demanda e a grande quantidade de parâmetros relacionados a processos físicos, químicos e biológicos.

É de fundamental importância a utilização de técnicas que venham a contribuir com uma gestão eficiente da água bruta, de forma a garantir que esta seja tratada e distribuída de modo eficaz e igualitário para as partes demandantes, levando em consideração as incertezas presentes no processo. Jägermeyr et al. (2016) destacam a importância de se adotar técnicas para otimizar as atividades relacionadas à gestão da água, considerando as particularidades locais de cada região, tornando, assim, as ferramentas de pesquisa operacional muito importantes no auxílio do problema em questão.

No trabalho de Scaratti, Michelon e Scaratti (2013), foi apresentado um modelo de avaliação da eficiência da gestão dos serviços municipais de abastecimento de água potável e de esgotamento, no qual utilizaram o método de Análise Envoltória de Dados (DEA - Data Envelopment Analysis). O estudo realizado considerou um total de 53 municípios com infraestrutura instalada para o abastecimento de água e esgotamento sanitário, considerando os seguintes indicadores de desempenho: a perspectiva de clientes, do mercado, conformidade dos produtos e a situação econômica financeira. Sob esses critérios, chegaram à conclusão que a maioria dos municípios avaliados apresentou uma gestão ineficiente.

Dentro da área de pesquisa operacional encontra-se a programação linear (PL) fuzzy, que tem sido muito utilizada nos últimos anos para tratar problemas que envolvam variáveis e funções, entre elas, com incertezas e imprecisões de várias naturezas, muitas de difícil quantificação, isto é, difusas ou nebulosas. Dentre esses, encontra-se o problema de alocação de água para vários usuários em ambientes com incertezas, como foi apresentado em Tsakiris e Spiliotis (2004). Essas relações são inerentes ao processo, pois tanto as receitas esperadas como as restrições, que necessariamente estão associadas a fatores sociais e ambientais, não poderiam ser determinadas de maneira precisa.

Canavese, Ortega e Giatti (2012) ressaltam que a lógica fuzzy precisa ser mais explorada, pois é uma ferramenta que possibilita a ação de uma análise em vários meios e que pode retratar a realidade presente no campo de saúde ambiental.

O presente trabalho apresenta um modelo de PL fuzzy para a distribuição de água para 15 zonas, na cidade de Campos, no norte-fluminense, considerando incertezas no parâmetro de demanda e consumo médio per capita. Esse modelo é previamente tratado como um problema de PL tradicional que servirá de base para introduzir as incertezas nos parâmetros, que será chamado de modelo crisp.

Um dos fatores que contribui para essas incertezas é o processo pelo qual a água necessita passar para que seja considerada ideal para consumo humano, pois ela, sem tratamento, pode causar doenças graves, ficando a cargo do Ministério da Saúde a fiscalização do fornecimento adequado do recurso (CESAN, 2013). Isso se justifica pelo fato de a água apresentar, geralmente, impurezas nos formatos gasoso e líquido, e poder ter, também, partículas sólidas, que estão ou não visíveis a olho nu (RECESA, 2008). Essa água é captada diretamente de rios e lagoas e é conhecida comumente como água doce, e tecnicamente como água bruta. Ao passar pelo processo que a torna adequada para o consumo da população, essa água passa a ser conhecida como água tratada.

Neste trabalho é apresentada, mais especificamente, a formulação de um modelo, por meio da ferramenta de pesquisa operacional conhecida como PL fuzzy, para alocação de água tratada à comunidade do 
município em questão. São considerados apenas os custos com produtos químicos utilizados no tratamento, de forma que se chegue à minimização destes, obtendo a solução ótima do modelo, considerando as incertezas presentes no processo de distribuição de água tratada nesse local. Essa distribuição é feita por uma empresa que fornece o serviço de abastecimento público e quem também disponibilizou publicamente informações importantes sobre esse abastecimento, que foram utilizadas neste trabalho para alimentar o modelo. A resolução numérica foi obtida rodando no software aberto e livre Rstudio. Com ele, calculou-se a solução ótima, considerando uma adaptação do pacote oferecido no trabalho de Villacorta et al. (2015).

\section{METODOLOGIA}

\section{Aspectos conceituais do tratamento de água}

A água, na sua apresentação natural, é chamada de água bruta, e pode ser potável ou não - no último caso, deve passar por algumas etapas de tratamento, que necessitam de produtos químicos para torná-la apta para o consumo. Esses produtos químicos podem ser utilizados em maior ou em menor quantidade. Isso depende da qualidade apresentada pela água captada das fontes. É de fundamental importância saber a qualidade da água bruta a ser tratada pelas estações responsáveis por esse serviço, pois essa qualidade irá servir de base para definir o tipo de tecnologia a ser utilizada para fins de adequação da água para abastecimento público (OLIVEIRA et al., 2014).

Quanto mais limpa é a água bruta, menor é a quantidade de substâncias químicas que são utilizadas no seu processo de tratamento. Porém, se essa água apresenta muitas impurezas, é necessária uma grande quantidade dessas substâncias para que se chegue à qualidade ideal para esse recurso, o que gera resíduos, como o lodo, que devem ser dispostos da forma correta (KATAYAMA et al., 2015).

Segundo Silva, Lucas e Teixeira (2012), o custo com produtos químicos é a segunda maior despesa na operação de uma estação de tratamento de água, representando $26 \%$ dos custos totais, sendo menor apenas que as despesas envolvendo recursos humanos, materiais e serviços.

Sousa, Monteiro e Silva (2012) comentam que o tratamento convencional é acompanhado de filtração direta, o processo mais utilizado para tratamento de água no Brasil. Esse processo tem um custo considerável, pois consiste em todas as fases de tratamento de água: coagulação, floculação, decantação, filtração, desinfecção, fluoretação e correção de pH. Esse processo é também conhecido como tratamento completo.

Além do tratamento convencional, pode ser feito um tratamento de água captada que apresente menos fases. Neste caso, é comum utilizar sempre as etapas de desinfecção e fluoretação (RECESA, 2008). Obviamente, esse tipo de tratamento, também conhecido como não convencional, é mais barato que o completo.
Esse fato está diretamente ligado ao custo necessário para o tratamento da água, porque quanto maior a quantidade de substâncias químicas aplicadas para o tratamento desse recurso, mais custoso será para a companhia de abastecimento hídrico, o que fará com que o custo seja repassado para o consumidor.

Segundo Cesan (2013) e Recesa (2008), a água pode passar por até sete etapas para que seja considerada apta para consumo. Essas etapas e suas respectivas descrições são as seguintes:

- Coagulação: tem por objetivo aglomerar as partículas de impurezas presentes na água de forma que se unam umas as outras, facilitando assim sua extração nas etapas posteriores. Para que isso ocorra, é necessário que se adicionem substâncias químicas na água, como o sulfato de alumínio, o cloreto de polialumínio (PAC), o sulfato férrico e o cloreto férrico;

- Floculação: nesta etapa, as partículas aglomeradas são processadas para que se formem flocos nos floculadores, que podem ser divididos em câmaras, mecânicos ou hidráulicos;

- Decantação: é um processo que visa à remoção dos flocos formados na etapa anterior, de forma que a água e os flocos sejam encaminhados para o tanque de decantação, no qual os flocos ficarão depositados no fundo, facilitando assim sua retirada;

- Filtração: tem por objetivo a remoção das partículas de impurezas que não foram extraídas na fase de decantação. Essa remoção ocorre por meio da formação de barreiras de areia, pedras e antrácito, submetendo assim a passagem da água por elas;

- Desinfecção: nesta etapa, são eliminados os microrganismos patogênicos que não foram retirados pelos processos anteriores. Para isso, adicionam-se sustâncias químicas na água, como o cloro gasoso, o hipoclorito de cálcio, o hipoclorito de sódio, o dióxido de cloro, o ozônio e a radiação ultravioleta;

- Fluoretação: é um processo que visa à proteção dos dentes contra as cáries, visto que as bactérias presentes nas placas dentárias produzem ácidos que deixam os dentes vulneráveis. Nesse processo, como o próprio nome já sugere, são adicionadas substâncias químicas à base de flúor, que podem ser o ácido fluossilícico e o fluorsilicato de sódio;

- Correção do pH: esta etapa deve ser realizada apenas quando for necessária, ou seja, quando o $\mathrm{pH}$ estiver fora do intervalo compreendido entre os limites 6 e 9,5. Neste caso, são adicionados produtos químicos para a realização dessa correção, que pode ser a cal virgem ou hidratada. Caso a água esteja dentro dos limites permitidos, essa correção não precisa ser feita.

\section{Programação linear fuzzy para alocação de água}

A abordagem do modelo de PL fuzzy assume que valores de determinados parâmetros ou coeficientes de um modelo de PL clássico sejam aceitos como os valores crisp, e que as restrições sejam modeladas por 
um conjunto fuzzy, que delimitará o grau de pertinência ou aproximação a ser aceita na verificação das restrições. Assim, a formulação pode ser vista na Equação 1:

otimizar $z=c^{T} x$

s.a. $\quad A x \widetilde{\leq} b$

$x \geq 0$

$x, c \in \mathfrak{N}^{n} ; A_{m \times n} ; b \in \mathfrak{R}^{m}$

Em que:

A restrição $\widetilde{\leq}$ representa o nível de aproximação ou a difusividade aceita para as restrições.

A abordagem de Zimmerman (1978), como apresentado em Fullér (2010), será utilizada para resolver o modelo PL fuzzy da Equação 1, que é solucionado via um modelo de PL por metas, estabelecendo um nível de aspiração para o valor $\mathrm{Z}$ da função objetivo.

A formulação do modelo incorpora a incerteza nos recursos e a estimativa prudente para o valor da função objetivo, ou considera-se alguma flexibilidade para a violação nas restrições incluindo no estimado de Z. Dessa forma, buscar-se-á uma solução $x^{\star}\left(x 1^{*}, x 2^{*}, \ldots, x n^{*}\right)$ que satisfaça as desigualdades da Equação 2 (FULLÉR, 2010):

$a_{01} x_{1}+\ldots+a_{0 n} x_{n} \leq\left(b_{0}, d_{0}\right)$

$a_{11} x_{1}+\ldots+a_{1 n} x_{n} \leq\left(b_{1}, d_{1}\right)$

$\vdots$

$a_{m 1} x_{1}+\ldots+a_{m n} x_{n} \leq\left(b_{m}, d_{m}\right)$

$l_{j} \leq x_{j} \leq u_{j} ; j=1, \ldots, n$

Em que:

Os parâmetros $\mathrm{a}_{01}, \mathrm{a}_{02}, \ldots, \mathrm{a}_{0 \mathrm{n}}$ são os coeficientes da função objetivo; os coeficientes $\mathrm{a}_{11}, \mathrm{a}_{12}, \ldots, a_{\mathrm{mn}}$ definem a matriz de coeficientes tecnológicos do modelo de PL clássico, ou o lado esquerdo das restrições do modelo;

$\mathrm{b}_{0}$ representa a meta que se aspira alcançar pela função objetivo;

$b_{1}, \ldots, b_{m}$ são os parâmetros do lado direito das restrições acompanhados com as amplitudes de imprecisão permitidas, representadas pelos valores $\mathrm{d}_{0}, \mathrm{~d}_{1}, \ldots, \mathrm{d}_{\mathrm{m}}$;

$l_{j}$ e $u_{j}$ são os limitantes inferior e superior das $x_{j}$, respectivamente.

Então, busca-se uma solução $x^{\star}$ que atenda às restrições em 2 num grau de satisfação aceitável, medida pela função de pertinência $0 \leq \mu_{j}\left(x^{*}\right) \leq 1, j=0,1, \ldots, m$; assim, a solução ao modelo PL fuzzy (2) deve satisfazer a maior condição de pertinência ou $\lambda^{*}=\max _{x} \min \left\{\mu_{0}(x), \mu_{1}(x), \ldots, \mu_{m}(x)\right\}$. Isto é, $\mathrm{em}^{*}$ verifica-se a maior satisfação para as restrições e o valor estimado de Z, o que se calcula via modelo de PL, como pode ser visto na Equação 3:

$$
\begin{array}{ll} 
& \max _{x} \quad \lambda \\
\text { s.a. } \quad & \mu_{0}(x) \geq \lambda \\
& \mu_{1}(x) \geq \lambda \\
& \vdots \\
& \mu_{m}(x) \geq \lambda \\
& 0 \leq \lambda \leq 1 ; x \in \mathfrak{R}^{n}
\end{array}
$$

A seguir, mencionam-se dois trabalhos que foram básicos para a presente pesquisa, que segue a recomendação de utilização de dados do mundo real, visando analisar as consequências tanto econômicas quanto ambientais relacionadas à alocação de água, buscando assim uma distribuição ótima dos recursos hídricos para seus múltiplos destinos (MORAES; CIRILO; SAMPAIO, 2004).

Tsakiris e Spiliotis (2004) desenvolveram um exemplo numérico simplificado para tratar o problema de alocação de água. Esse modelo possui seis nós contendo superfície de água e dois centros de consumo deste recurso. No modelo proposto foi feita uma simulação que teve como objetivo buscar um retorno econômico que fosse o mais alto possível e, ao mesmo tempo, garantir a quantidade mínima de água requerida a todas as áreas demandantes pelo recurso.

Rodella (2014) desenvolveu um modelo de PL para determinar a quantidade ótima de água distribuída para 12 municípios da região metropolitana do Recife, visando minimizar uma parte significativa dos custos de uma empresa de abastecimento de água, constituída pela soma do custo de energia elétrica e dos custos de tratamento químico da água bruta utilizada. O modelo desenvolvido representou o abastecimento de água para $51 \%$ da população do estado de Pernambuco, em 2013, e teve como parâmetros da função objetivo o preço, por metro cúbico, da energia elétrica consumida no processo de captação, o tratamento e a distribuição da água na região, além do custo dos produtos químicos por metro cúbico de água tratada.

O presente trabalho apresenta um modelo genérico de PL fuzzy para o fornecimento de água da região metropolitana do município de Campos dos Goytacazes. Esse modelo leva em consideração os custos com produtos químicos, utilizados nos processos de tratamento de água, e sua técnica de solução estabelece a quantidade de água a se distribuir para cada grupo de bairros em que o município está dividido, de modo a ter o custo mínimo. As restrições dizem respeito às limitações presentes no fornecimento hídrico de cada sistema, levando em consideração os diferentes tipos de fontes hídricas disponíveis para atender às demandas de sua respectiva comunidade.

\section{Modelo para Campos dos Goytacazes}

O município de Campos dos Goytacazes situa-se no norte do estado do Rio de Janeiro, região norte-fluminense. É estrategicamente muito importante para o país, devido a sua alta participação na indústria de petróleo e gás, reconhecido como responsável por $80 \%$ da produção 
nacional de petróleo. O município, assim como dois terços do estado do Rio de Janeiro, é banhado pelo rio Paraíba do Sul (PERHI-RJ, 2014).

O sistema de abastecimento de água no município em questão é gerido pela concessionária Águas do Paraíba (ÁGUAS DO PARAÍBA, 2015). Segundo ela, o abastecimento de água da cidade é feito por 15 sistemas, fornecendo uma vazão média de 1.447 litros por segundo $\left(\right.$ L.s $\left.{ }^{-1}\right)$.

Cada sistema abastece um conjunto de bairros e cada bairro é abastecido apenas por um sistema, que é uma característica que torna simples a relação de abastecimento, diferentemente de outras regiões. $\mathrm{O}$ rio Paraíba do Sul abastece apenas um dos sistemas, porém este é o sistema de maior vazão, além de atender ao maior número de bairros da cidade. Os demais sistemas são abastecidos pela água de uma bateria de poços, rios menores, lagoas e córregos, como pode ser observado no Quadro 1.

Dados do Instituto Brasileiro de Geografia e Estatística (IBGE, 2008) mostram que o volume de água distribuída diariamente no município — no período da pesquisa - é de cerca de $70.000 \mathrm{~m}^{3}$, sendo aproximadamente $94 \%$ água tratada. Com relação a esta água tratada, cerca de $88 \%$ é distribuída após passar por tratamento convencional, enquanto que o restante passa por tratamento não convencional. O Quadro 1 mostra também a relação entre os sistemas de abastecimento com seus respectivos tipos de tratamento e quantidade de moradores atendidos.

Sabendo das características particulares relacionadas ao fornecimento hídrico em Campos dos Goytacazes, o presente trabalho buscou desenvolver um modelo genérico e simples que representasse essa dinâmica de distribuição de forma a se avaliar, por meio da PL fuzzy, o custo de abastecimento de água para o município de Campos dos Goytacazes, levando em consideração apenas os produtos químicos utilizados no tratamento da água bruta, que a converte em apropriada para o consumo humano. Sendo assim, utilizou-se o software Rstudio para obter os valores da simulação do modelo formulado.

Como visto anteriormente, o processo de tratamento da água utiliza produtos químicos de forma a torná-la potável. Esses produtos formam uma parcela importante dos custos de uma estação de tratamento de água. A utilização desses produtos vai depender do tipo de água bruta captada, pois quanto mais impurezas o líquido contiver mais produtos químicos serão utilizados para seu tratamento. Esses produtos são usados nas fases de coagulação, desinfecção e fluoretação. Na etapa de correção de $\mathrm{pH}$, a utilização do produto químico é opcional, caso seja necessária a correção desse fator.

Inicialmente, formula-se um modelo de PL padrão, buscando minimizar os custos mensais com produtos químicos na cidade de Campos dos Goytacazes, levando em consideração a quantidade mínima de água a ser distribuída à população, a quantidade máxima que ela consome e a capacidade de tratamento que o conjunto de estações pode oferecer.

\section{Antecedentes da formulação}

O primeiro passo para a obtenção do modelo é a formulação da função objetivo. Esta é composta do somatório dos custos decorrentes do volume de água a ser tratada em cada sistema em questão. Sendo assim, a definição das variáveis de decisão - enumeradas de 1 a 15 - está associada aos sistemas de abastecimento, como pode ser visto no Quadro 2. É importante ressaltar que esses custos estão associados às etapas de coagulação, desinfecção, fluoretação e, casualmente, correção de pH.

O modelo do presente trabalho adota a utilização de produtos químicos apenas nas fases em que a utilização desses é certa, ou seja, não

Quadro 1 - Características dos sistemas de abastecimento de Campos dos Goytacazes.

\begin{tabular}{|c|c|c|c|c|}
\hline Sistema & Tipo de tratamento & Quantidade de habitantes & Capacidade L.s ${ }^{-1}$ & Captação \\
\hline Beco de Santo Antônio & Não convencional & 15.000 & 40 & Poço Profundo \\
\hline Boa Vista & Não convencional & 25.000 & 60 & Poço Profundo \\
\hline Conselheiro Josino & Convencional & 1.500 & 3 & Córrego da Penha \\
\hline Coroa & Convencional & 350.000 & 1.200 & Rio Paraíba do Sul \\
\hline Donana & Não convencional & 25.000 & 70 & Poço Profundo \\
\hline Macabu & Não convencional & 1.300 & 3 & Poço Profundo \\
\hline Morangaba & Convencional & 1.000 & 5 & Rio Preto \\
\hline Morro do Coco & Não convencional & 1.000 & 2 & Poço Profundo \\
\hline Murundu & Não convencional & 500 & 1 & Poço Profundo \\
\hline Ponta Grossa & Convencional & 2.000 & 3 & Lagoa Feia \\
\hline Santo Eduardo/Santa Maria & Convencional & 6.000 & 20 & Poço Profundo \\
\hline São Sebastião & Não convencional & 4.000 & 15 & Poço Profundo \\
\hline Saturnino Braga & Não convencional & 3.500 & 15 & Poço Profundo \\
\hline Três Vendas & Convencional & 1.000 & 5 & Rio Muriaé \\
\hline Vila Nova & Não convencional & 1.200 & 5 & Poço Profundo \\
\hline
\end{tabular}


é considerada, neste modelo simplificado, a eventual adição de produtos químicos da fase de correção de $\mathrm{pH}$.

Para a etapa de coagulação, o produto químico mais utilizado é o sulfato de alumínio (RECESA, 2008). A quantidade a ser utilizada que apresenta um resultado satisfatório é de $25 \mathrm{mg}$ para cada litro de água bruta (NUNES, 2011). O custo referente a esse produto, segundo Adasa (2010), é de R\$ 0,52 por quilo. Dessa forma, tem-se estabelecido o custo da etapa de coagulação: $\mathrm{R} \$ 0,000013$ por litro.

$\mathrm{Na}$ fase de desinfecção, é utilizado com frequência o cloro gasoso. A quantidade deste produto não deve ser inferior a $0,2 \mathrm{mg}$ para cada litro de água (CESAN, 2013). Esse produto tem um custo, por quilo, de $\mathrm{R} \$ 3,93$ (ADASA, 2010). Sendo assim, o custo da etapa de desinfecção fica ajustado em $\mathrm{R} \$ 0,0000007$ por litro.

Para a fluoretação, segundo Cesan (2013), utiliza-se o ácido fluossilícico, com uma quantidade que, no Brasil, não é inferior a 0,6 mg para cada litro de água. Segundo Adasa (2010), o custo desse produto é de $\mathrm{R} \$ 0,58$ por quilo. Dessa forma, o custo da etapa de fluoretação, por litro, resulta em $\mathrm{R} \$ 0,000000348$.

De posse dos custos das etapas, pode-se estipular o custo por litro para o tratamento da água no sistema convencional e no não convencional. $\mathrm{O}$ custo do primeiro resulta em $\mathrm{R} \$ 0,000014$, que consiste na soma dos custos das etapas de coagulação, desinfecção e fluoretação. Já o custo do não convencional resulta em $\mathrm{R} \$ 0,000001$, obtido por meio da soma dos custos das etapas de desinfecção e fluoretação.

É importante ressaltar que os valores considerados para os custos não necessariamente correspondem aos valores atuais dos produtos químicos, visto que a extração foi feita baseada em Adasa (2010), porém representam uma estimativa coerente para a formulação de um modelo.

Quadro2- Relação entre variáveis de decisão e sistemas de abastecimento.

\begin{tabular}{|c|c|}
\hline Variável de decisão & Sistema \\
\hline$x_{1}$ & Beco de Santo Antônio \\
\hline$x_{2}$ & Boa Vista \\
\hline$x_{3}$ & Conselheiro Josino \\
\hline$x_{4}$ & Coroa \\
\hline$x_{5}$ & Donana \\
\hline$x_{6}$ & Macabu \\
\hline$x_{7}$ & Morro do Coco \\
\hline$x_{8}$ & Murundu \\
\hline$x_{9}$ & Ponta Grossa \\
\hline$x_{10}$ & Santo Eduardo/Santa Maria \\
\hline$x_{11}$ & São Sebastião \\
\hline$x_{12}$ & Saturnino Braga Vendas \\
\hline$x_{13}$ & Tila Nova \\
\hline$x_{14}$ & $x_{15}$ \\
\hline
\end{tabular}

Após a determinação dos parâmetros da função objetivo, é preciso estabelecer as restrições. No caso do modelo proposto por este trabalho, foram levados em consideração a quantidade total de água tratada nas formas convencional e não convencional; a quantidade mínima de água a ser fornecida para determinada comunidade, por meio de cada uma das quinze estações de tratamento; e a capacidade de tratamento de água que cada estação pode ofertar, totalizando assim 32 restrições.

Todas as restrições formuladas para o modelo de Campos dos Goytacazes obedecem à estrutura apresentada na Equação 2. Dessa forma, as restrições descritas na Equação 4 apresentam a característica de serem relações tecnológicas. Diferentemente do que acontece com as 30 restrições seguintes (Equação 5), que fornecem os limites individuais de cada uma das 15 variáveis e não apresentam essa característica.

As duas primeiras restrições estão relacionadas à quantidade mensal de água tratada que é distribuída nas formas convencional e não convencional (IBGE, 2008), limitando inferiormente o valor total distribuído aos municípios nas duas modalidades consideradas.

Para a quantidade mínima de água fornecida à determinada comunidade, leva-se em consideração os dados de Barbosa (2015), que informa que o mínimo diário de água que cada ser humano necessita para as suas atividades básicas é $110 \mathrm{~L}$. Sabendo-se também a quantidade de habitantes que cada estação de tratamento atende (Quadro 1), pode-se estimar o valor do consumo mínimo mensal de água a ser fornecido por cada estação de tratamento, mediante ao produto entre o mínimo diário humano; a quantidade de habitantes atendidos pela estação; e a quantidade de dias de um mês, estabelecendo assim as 15 restrições seguintes.

Para se estipular a capacidade mensal de produção de cada estação de tratamento de água, leva-se em consideração o produto entre a capacidade, em L.s ${ }^{-1}$, de cada estação (Quadro 1) e a quantidade de segundos contidos em um mês, estabelecendo assim as 15 últimas restrições.

Estabelecidos todos os antecedentes, pode-se formular o modelo de PL padrão para o caso de distribuição de água para o município de Campos dos Goytacazes.

\section{Modelo clássico de programação linear para o problema}

Levando em consideração os 15 sistemas de abastecimento e seus respectivos custos associados à utilização de produtos químicos, descritos na seção anterior, chega-se à seguinte formulação da função objetivo:

minimizar

$0,000001 x_{1}+0,000001 x_{2}+0,000014 x_{3}+0,000014 x_{4}+0,000001 x_{5}+0,0000$ $014 x_{6}+0,000014 x_{7}+0,000001 x_{8}+0,000001 x_{9}+0,000014 x_{10}+0,000014 x_{11}$ $+0,000001 x_{12}+0,000001 x_{13}+0,000014 x_{14}+0,000001 x_{15}$

De igual modo, podem-se formular as restrições do volume de água bruta tratada de forma convencional e não convencional na Equação 4, e na Equação 5, a capacidade mensal de produção e a quantidade mínima. 
$x_{3}+x_{4}+x_{7}+x_{10}+x_{11}+x_{14} \geq 1741470$

$x_{1}+x_{2}+x_{5}+x_{6}+x_{8}+x_{9}+x_{12}+x_{13}+x_{15} \geq 247290000$

$\begin{array}{lll}x_{1} \geq 49500000 & x_{2} \geq 82500000 & x_{3} \geq 4950000 \\ x_{4} \geq 1155000000 & x_{5} \geq 82500000 & x_{6} \geq 4290000 \\ x_{7} \geq 3300000 & x_{8} \geq 3300000 & x_{9} \geq 1650000 \\ x_{10} \geq 6600000 & x_{11} \geq 19800000 & x_{12} \geq 13200000 \\ x_{13} \geq 11550000 & x_{14} \geq 3300000 & x_{15} \geq 3960000 \\ x_{1} \leq 103680000 & x_{2} \leq 155520000 & x_{3} \leq 7776000 \\ x_{4} \leq 3110400000 & x_{5} \leq 181440000 & x_{6} \leq 7776000 \\ x_{7} \leq 12960000 & x_{8} \leq 5184000 & x_{9} \leq 2592000 \\ x_{10} \leq 7776000 & x_{11} \leq 51840000 & x_{12} \leq 38880000 \\ x_{13} \leq 38880000 & x_{14} \leq 12960000 & x_{15} \leq 12960000\end{array}$

\section{RESULTADOS E DISCUSSÃO}

Após a formulação do modelo crisp ter sido feita, na seção "Modelo clássico de programação linear para o problema”, foi realizada a simulação, utilizando o software R, para que se chegasse ao custo mensal de fornecimento de água relacionado aos produtos químicos para o município de Campos dos Goytacazes e aos volumes alocados de cada sistema de abastecimento para suas respectivas comunidades demandantes. $\mathrm{O}$ software $\mathrm{R}$ possui um pacote para o algoritmo Simplex com uma precisão numérica aceitável. O apêndice apresenta a formulação do modelo proposto no software utilizado.

Com relação ao custo, chegou-se a um valor mensal com produtos químicos de $\mathrm{R} \$ 24.634,75$, que representa o custo total mínimo entre todas as estações de tratamento da cidade de Campos dos Goytacazes para atendendo de todas as comunidades. Para a quantidade de água fornecida pelos sistemas de abastecimento, chegou-se aos valores, em litros, observados no Quadro 3.

Os resultados mostraram que o modelo de PL crisp foi satisfatório, mesmo com algumas variáveis apresentando valores de restrição, como $x_{7}, x_{12}$ e $x_{15}$. Porém, o conceito de PL padrão não permite a cobertura de incertezas que o problema possa apresentar. É o que acontece neste caso com as duas primeiras restrições, que tratam da quantidade total de água fornecida nas formas convencional e não convencional. Os valores dessas restrições foram limitados inferiormente pelo valor fornecido pelo IBGE (2008). Porém, esses valores sofrem variação mediante a demanda de água das comunidades.
Sendo assim, pode-se recorrer a um modelo de PL fuzzy que permite tratar de problemas que envolvem incertezas, o que será feito a seguir.

\section{O modelo de programação linear fuzzy para o problema}

Uma segunda versão do modelo de alocação de água para a cidade de Campos dos Goytacazes tem o objetivo de considerar as imprecisões encontradas na dinâmica do sistema, de forma que se possa ter uma representação mais fiel do que de fato ocorre na prática. Essa característica imprecisa é inserida por meio do componente fuzzy da PL por metas, referida na Equação 2, de forma que as duas primeiras restrições, que dizem respeito à quantidade de água distribuída nas formas convencional e não convencional, permitam incertezas, pois essa quantidade certamente vai variar dependendo da demanda do mês.

Sendo assim, foi calculado o modelo de PL por metas fuzzy considerando um relaxamento de $10 \%$ no lado direito das duas restrições iniciais, tendo assim, por meta, um valor $10 \%$ mais baixo que o valor encontrado no modelo anterior, em que não se incorporava o componente fuzzy. O Apêndice traz detalhes do modelo implementado no "R". Esses valores adotados para as tolerâncias das restrições e para a meta foram definidos mediante ao conhecimento sobre o processo, de forma que outras mudanças possam ser feitas, de acordo com o objetivo que se pretende.

Feitas as mudanças, chegou-se a um valor para a função objetivo de $\mathrm{R} \$ 22.220,78$, que representa uma diminuição de 9,8\% dos custos totais com produtos químicos para a cidade de Campos dos Goytacazes em relação ao modelo anterior, que não considerava as variações que poderiam ocorrer no lado direito de algumas restrições. Para a quantidade alocada pelos sistemas de abastecimento, chegou-se aos valores dispostos no Quadro 4 .

A principal mudança ocorreu na variável $x_{4}$, que sofreu um decréscimo no valor de seu fornecimento, possibilitando assim a diminuição no custo total do sistema.

Dessa forma, pode-se observar que o modelo de PL fuzzy cumpriu o objetivo proposto, que foi o de poder incluir as imprecisões pertencentes ao processo de distribuição de água. Isso fez com que a dinâmica do sistema de abastecimento de água fosse representada de forma mais ajustada ao que acontece na pratica, pois a abordagem utilizando lógica fuzzy incorpora as variações inerentes aos processos mais complexos, o que não é possível em abordagens com a PL padrão.

Quadro 3 - Variáveis de decisão do modelo proposto: quantidade mensal fornecida, em litros.

\begin{tabular}{|l|c|c|c|c|c|}
\hline Variáveis & $\mathrm{x}_{1}$ & $\mathrm{x}_{2}$ & $\mathrm{x}_{3}$ & $\mathrm{x}_{4}$ & $\mathrm{x}_{5}$ \\
\hline Resultados & 49500000 & 82500000 & 7776000 & 1700694000 & 82500000 \\
\hline Variáveis & $\mathrm{x}_{6}$ & $\mathrm{x}_{7}$ & $\mathrm{x}_{8}$ & $\mathrm{x}_{9}$ & $\mathrm{x}_{10}$ \\
\hline Resultados & 4290000 & 3300000 & 3300000 & 1650000 & 6600000 \\
\hline Variáveis & $\mathrm{x}_{11}$ & $\mathrm{x}_{12}$ & $\mathrm{x}_{13}$ & $\mathrm{x}_{14}$ & $\mathrm{x}_{15}$ \\
\hline Resultados & 19800000 & 13200000 & 11550000 & 3300000 & 3960000 \\
\hline
\end{tabular}


Quadro 4 - Variáveis de decisão do modelo fuzzy proposto: quantidade mensal fornecida, em litros.

\begin{tabular}{|c|c|c|c|c|c|}
\hline Variáveis & $x_{1}$ & $x_{2}$ & $x_{3}$ & $x_{4}$ & $x_{5}$ \\
\hline Resultados & 49500000 & 82500000 & 7776000 & 1528267921 & 82500000 \\
\hline Variáveis & $x_{6}$ & $x_{7}$ & $x_{8}$ & $x_{9}$ & $x_{10}$ \\
\hline Variáveis & $x_{11}$ & $x_{12}$ & $x_{13}$ & $x_{14}$ & $x_{15}$ \\
\hline Resultados & 19800000 & 13200000 & 11550000 & 3300000 & 3960000 \\
\hline
\end{tabular}

\section{CONCLUSÕES}

Uma boa gestão da água doce e saneamento adequado são essenciais para o bem-estar humano e o desenvolvimento sustentável. O Brasil adotou a Agenda 2030 das Nações Unidas, em 2015, que propõe em seu sexto objetivo "Assegurar a disponibilidade e gestão sustentável da água e saneamento para todos". A fim de atingir esse objetivo, deve ser considerado o fator de disponibilidade da água, o que exige maior preocupação no gerenciamento que garantirá sua distribuição de qualidade e o uso adequado.

A água doce no planeta apresenta muitas incertezas, tanto em relação à oferta quanto à demanda, pois condições climáticas e ambientais as afetam fortemente. Sendo assim, os responsáveis pela tomada de decisão para a gestão necessitam de ferramentas que possam incorporar essas incertezas. A lógica difusa, ou fuzzy, torna-se muito importante para esse fim, pois ajudará na modelagem de sistemas que apresentam essa característica, fazendo com que a resposta resultante seja a mais confiável possível.

O modelo desenvolvido por este trabalho mostrou uma abordagem experimental, levando em consideração os custos nos sistemas de abastecimento de água no município de Campos dos Goytacazes relacionados aos produtos químicos utilizados no tratamento da água, mostrando como a abordagem difusa pode inserir um caráter impreciso ao modelo. Os resultados mostraram que simples variações podem produzir resultados significativamente diferentes. Pode-se ver que a inserção do caráter difuso nas duas últimas restrições fez com que o modelo nos fornecesse um resultado $9,8 \%$ menor que a simulação feita por PL clássica, o que mostra a eficiência dessa ferramenta para o tipo de problema abordado.

Sendo assim, este modelo servirá de ponto de partida para uma abordagem mais criteriosa do sistema de abastecimento de água de Campos dos Goytacazes, visto que os valores adotados para os custos se embasaram no que a literatura fornece sobre tratamento químico, e a modelagem desenvolvida utilizou dados encontrados no portal eletrônico da distribuidora de águas do município. Porém, trabalhos futuros poderão extrair informações mais precisas da própria empresa distribuidora, de forma a tornar o modelo mais ajustado, fornecendo, por exemplo, valores mais exatos dos custos pagos em Campos dos Goytacazes. $\mathrm{O}$ modelo aqui proposto atendeu às expectativas, pois permitiu formular e computar a solução do modelo relacionado a um problema de custos com o tratamento químico na alocação de água de um município. O modelo utilizado foi o de PL fuzzy, que permitiu tratar as incertezas devido a tolerâncias aceitáveis nas restrições e avaliar essa abordagem flexibilizada do modelo clássico de PL, por meio do algoritmo simplex, apresentando resultados satisfatórios. Essa metodologia proposta tem uma relação direta e positiva com a racionalização da água bruta, pois uma gestão eficiente da água tratada, por parte da administração pública, garantirá uma distribuição mais uniforme aos usuários e com o menor custo.

\section{REFERÊNCIAS}

ACSELRAD, M.V:; AZEVEDO, J.P.S.; JOHNSSON, R.S.M. (2015) Cobrança pelo uso da água no Estado do Rio de Janeiro, Brasil (2004-2013): histórico e desafios atuais. Revista Engenharia Sanitária e Ambiental, v. 20, n. 2, p. 199-208. DOI: 10.1590/S1413-41522015020000112026

AGÊNCIA REGULADORA DE ÁGUAS, ENERGIA E SANEAMENTO BÁSICODO DISTRITOFEDERAL (ADASA). (2O10) Proposta referente à primejra revisão tarifária periódica das tarifas do serviço público de abastecimento de água e esgotamento sanitário praticadas pela CAESB. Adasa.
AGUAS DO PARAÍBA. GRUPO ÁGUAS DO BRASIL (2015) Estação de tratamento de água. Disponível em: <https://www. grupoaguasdobrasil.com.br/aguas-paraiba/agua-e-esgoto/ estacao-tratamento-agua/>. Acesso em: jun. 2018.

BARBOSA, V. (2015) 20 números revelam o drama da água no mundo. Exame, 22 março 2015. Disponível em: <https://exame. abril.com.br/mundo/20-numeros-alarmantes-sobre-a-agua-nomundo/>. Acesso em: jun. 2018. 
CANAVESE, D.; ORTEGA, N.R.S.; GIATTI, L.L. (2012) Abordagem ecossistêmica e lógica Fuzzy: uma proposta dialética para o uso da informação em Saúde Ambiental. Engenharia Sanitária e Ambiental, v. 17, n. 4, p. 363-368.

CARVALHO, M.A.; MELLO JÚNIOR, A.V.M.; SCHARDONG, A.; PORTO, R.L.L. (2009) Sistema de suporte à decisão para alocação de água em projetos de irrigação. Revista Brasileira de Engenharia Agrícola e Ambiental, Campina Grande, v. 13, n. 1, p. 10-17. DOI: 10.1590/S141543662009000100002

CHAIB, E.B.; RODRIGUES, F.C.; MAIA, B.H.; NASCIMENTO, N.D.O. (2O15) Avaliação do potencial de redução do consumo de água potável por meio da implantação de sistemas de aproveitamento de água de chuva em edificações unifamiliares. Revista Brasileira de Recursos Hídricos, Porto Alegre, v. 20, n. 3, p. 605-614. DOI: 10.21168/rbrh.v2On3.p605-614

COMPANHIA ESPÍRITO SANTENSE DE SANEAMENTO (CESAN). (2013) Apostila de tratamento da água. Disponível em: <http://www. cesan.com.br>. Acesso em: abr. 2016.

FULLÉR, R. (2010) An Introduction to Fuzzy Linear Programs Tutorial. Disponível em: <http://uni-obuda.hu/users/fuller.robert/ linear.pdf>. Acesso em: mar. 2016.

INSTITUTO BRASILEIRO DE GEOGRAFIA E ESTATÍSTICA (IBGE). (2008) Pesquisa Nacional de Saneamento Básico. Disponível em: <https://cidades.ibge.gov.br/brasil/rj/campos-dos-goytacazes/ pesquisa/30/30051>. Acesso em: jun. 2016.

JÄGERMEYR, J.; GERTEN, D.; SCHAPHOFF, S.; HEINK, J.; LUCHT, W.; ROCKSTRÖM, J. (2016) Integrated crop water management might sustainably halve the global food gap. Environmental Research Letter, v. 11, n. 2. Disponível em: <http://iopscience.iop.org/ article/10.1088/1748-9326/11/2/025002/pdf>. Acesso em: jun. 2016. DOI: 10.1088/1748-9326/11/2/025002

KATAYAMA, V.T.; MONTES, C.P.; FERRAZ, T.H.; MORITA, D.M. (2O15) Quantificação da produção de lodo de estações de tratamento de água de ciclo completo: uma análise crítica. Engenharia Sanitária e Ambiental, v. 20, n. 4, p. 559-569.

MORAES, M.G.A.; CIRILO, J.A.; SAMPAIO, Y. (2004) Integração dos componentes econômico e hidrológico na modelagem de alocação ótima de água para apoio a gestão de recursos hídricos: uma aplicação na bacia do Rio Pirapama. In: ENCONTRO NACIONAL DE ECONOMIA, 32. Anais...

NUNES, M.L.A. (2011) Avaliação da eficiência de diferentes produtos químicos utilizados na coagulação da água. (Trabalho de conclusão do curso de Engenharia Ambiental) - Universidade Federal de Rondônia, Rondônia.

OLIVEIRA, M.D.; REZENDE, O.L.T.; OLIVEIRA, S.M.A.C.; LIBÂNIO, M. (2O14) Nova abordagem do Índice de Qualidade de Água Bruta utilizando a Lógica Fuzzy. Engenharia Sanitária e Ambiental, v. 19, n. 4, p. 361-372.

PAULINO, M.P. (2005) Alternativas de Concepção e Gestão de Gestão de Abastecimento de Águas de Cidades que Utilizam
Vários Mananciais: Caso Ouro Preto/MG. Dissertação (Mestrado) Universidade Federal de Ouro Preto, Ouro Preto.

PLANO ESTADUAL DE RECURSOS HÍDRICOS DO ESTADO DO RIO DE JANEIRO (PERHI-RJ). (2014). Relatório Gerencial. Rio de Janeiro: INEA/RJ. Disponível em: <http://www.inea.rj.gov.br/cs/groups/ public/documents/document/zwew/mdcx/ edisp/inea0071538. pdf>. Acesso em: abr. 2017.

REDE NACIONAL DE CAPACITAÇÃO E EXTENSÃO TECNOLÓGICA EM SANEAMENTO AMBIENTAL (RECESA). (2008) Abastecimento de água: operação e manutenção de estações de tratamento de água: guia do profissional em treinamento: nível 2. Belo Horizonte: Ministério das Cidades / Secretaria Nacional de Saneamento Ambiental.

RODELLA, L.M.A. (2014) Modelo de programação linear para apoio a decisão na distribuição de água de sistemas integrados de abastecimento. Tese (Doutorado) - Universidade Federal do Pernambuco, Recife.

SCARATTI, D:; MICHELON, W:; SCARATTI, G. (2013) Avaliação da eficiência da gestão dos serviços municipais de abastecimento de água e esgotamento sanitário utilizando Data Envelopment Analysis. Revista Engenharia Sanitária e Ambiental, v. 18, n. 4, p. 333-340.

SILVA, L.; LUCAS, H.; TEIXEIRA, M.R. (2012) Avaliação econômica preliminar da nanofiltração na remoção de cianotoxinas em água naturais. Sapientia.

SISTEMA NACIONAL DE INFORMAÇÕES SOBRE SANEAMENTO (SNIS). (2016) Diagnóstico dos Serviços de Água e Esgotos - 2014. Brasília: Secretaria Nacional de Saneamento Ambiental/Ministério das Cidades. 212 p.

SOUSA, L.R.; MONTEIRO, S.J.; SILVA, W.O. (2012) Tratamento de água para abastecimento. Universidade Regional do Cariri. CCT. Juazeiro do Norte, Ceará.

TSAKIRIS, G.; SPILIOTIS, M. (2004) Fuzzy linear Programming for problems of water allocation under uncertainty. European Water. v. 7-8, p. 25-37.

TUNDISI, J.G. (2008) Recursos hídricos no futuro: problemas e soluções. Estudos Avançados, v. 22, n. 63. DOI: 10.1590/SO10340142008000200002

VILLACORTA, P.J.; RABELO, C.A.; PELTA, D.A.; VERDEGAY, J.L. FuzzY LP: an R Package for Solving Fuzzy Linear Programming Problems. Departamento de Ciências da Computação e Inteligência Artificial. Espanha, Universidade de Granada. Disponível em: <https:// cran.r-project.org/web/packages/FuzzyLP/vignettes/FuzzyLP.pdf> Acesso em: out. 2015.

WORLD WIDE FUND FOR NATURE (WWF). (2014) Planeta Vivo Relatório 2014. Disponível em: <http://d3nehc6yl9qzo4.cloudfront. net/downloads/relatorio_planeta_vivo2014_sumario.pdf>. Acesso em: out. 2015. 
Apêndice - Formulações do modelo proposto utilizando o "r".

Esta seção teve como objetivo apresentar o código desenvolvido no ambiente R para formular o problema proposto no trabalho para alocação de água no município de Campos dos Goytacazes.

O vetor "f.obj" fornece os valores dos custos de cada sistema de abastecimento. A matriz "f.con" fornece os coeficientes das restrições do modelo. O termo "f.dir" apresenta a direção da desigualdade associada a cada restrição, podendo esta ser "maior ou igual" ou "menor ou igual". O vetor "f.rhs" fornece os valores do lado direito associado a cada restrição. E, ao final, a função "lp" calcula a solução de programação linear padrão para o problema em questão. O código implementado possui a seguinte configuração:

f.obj <- c $(0.000001,0.000001,0.000014,0.000014,0.000001,0.0000014,0.000014,0.000001,0.000001,0.000014,0.000014,0.000001$, $0.000001,0.000014,0.000001)$

f.con <- matrix $(\mathrm{c}(0,0,1,1,0,0,1,0,0,1,1,0,0,1,0$,

$1,1,0,0,1,1,0,1,1,0,0,1,1,0,0$

$1,0,0,0,0,0,0,0,0,0,0,0,0,0,0$,

$0,1,0,0,0,0,0,0,0,0,0,0,0,0,0$,

$0,0,1,0,0,0,0,0,0,0,0,0,0,0,0$,

$0,0,0,1,0,0,0,0,0,0,0,0,0,0,0$,

$0,0,0,0,1,0,0,0,0,0,0,0,0,0,0$,

$0,0,0,0,0,1,0,0,0,0,0,0,0,0,0$,

$0,0,0,0,0,0,1,0,0,0,0,0,0,0,0$,

$0,0,0,0,0,0,0,1,0,0,0,0,0,0,0$,

$0,0,0,0,0,0,0,0,1,0,0,0,0,0,0$,

$0,0,0,0,0,0,0,0,0,1,0,0,0,0,0$,

$0,0,0,0,0,0,0,0,0,0,1,0,0,0,0$,

$0,0,0,0,0,0,0,0,0,0,0,1,0,0,0$,

$0,0,0,0,0,0,0,0,0,0,0,0,1,0,0$,

$0,0,0,0,0,0,0,0,0,0,0,0,0,1,0$,

$0,0,0,0,0,0,0,0,0,0,0,0,0,0,1$,

$1,0,0,0,0,0,0,0,0,0,0,0,0,0,0$,

$0,1,0,0,0,0,0,0,0,0,0,0,0,0,0$,

$0,0,1,0,0,0,0,0,0,0,0,0,0,0,0$,

$0,0,0,1,0,0,0,0,0,0,0,0,0,0,0$,

$0,0,0,0,1,0,0,0,0,0,0,0,0,0,0$,

$0,0,0,0,0,1,0,0,0,0,0,0,0,0,0$,

$0,0,0,0,0,0,1,0,0,0,0,0,0,0,0$,

$0,0,0,0,0,0,0,1,0,0,0,0,0,0,0$,

$0,0,0,0,0,0,0,0,1,0,0,0,0,0,0$,

$0,0,0,0,0,0,0,0,0,1,0,0,0,0,0$,

$0,0,0,0,0,0,0,0,0,0,1,0,0,0,0$,

$0,0,0,0,0,0,0,0,0,0,0,1,0,0,0$,

$0,0,0,0,0,0,0,0,0,0,0,0,1,0,0$,

$0,0,0,0,0,0,0,0,0,0,0,0,0,1,0$,

$0,0,0,0,0,0,0,0,0,0,0,0,0,0,1)$, nrow $=32$, byrow=TRUE)

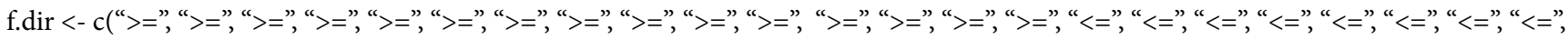
“<=", “<=", “<=", “<=", “<=", “<=", “<=", “>=”, “>=")

f.rhs <- c (49500000, 82500000, 4950000, 1155000000, 82500000, 4290000, 3300000, 3300000, 1650000, 6600000, 19800000, 13200000, $11550000,3300000,3960000,103680000,155520000,7776000,3110400000,181440000,7776000,12960000,5184000,2592000,7776000$, $51840000,38880000,38880000,12960000,12960000,1741470000,247290000)$

lp (“min”, f.obj, f.con, f.dir, f.rhs)

lp (“min”, f.obj, f.con, f.dir, f.rhs)\$solution

Para a simulação do modelo fuzzy, substituiu-se as duas últimas linhas do código, colocando em seu lugar o vetor de tolerâncias (t) e a função fuzzy, de acordo com a proposta do modelo. Sendo assim, as duas últimas linhas do código dessa seção deram lugar a:

$\mathrm{t}<-\mathrm{c}(0,0,0,0,0,0,0,0,0,0,0,0,0,0,0,0,0,0,0,0,0,0,0,0,0,0,0,0,0,0,174147000,24729000)$

FCLP.fuzzyObjective(f.obj, f.con, f.dir, f.rhs, $t, z 0=22171.275, t 0=50, F)$ 